\title{
ON STRUCTURE SPACES OF IDEALS IN RINGS OF CONTINUOUS FUNCTIONS
}

BY

\section{DAVID RUDD}

\begin{abstract}
ABSTR ACT. A ring of continuous functions is a ring of the form $C(X)$, the ring of all continuous real-valued functions on a completely regular Hausdorff space $X$.

With each ideal $I$ of $C(X)$, we associate certain subalgebras of $C(X)$, and discuss their structure spaces.

We give necessary and sufficient conditions for two ideals in rings of continuous functions to have homeomorphic structure spaces.
\end{abstract}

Introduction. For a subset $A$ of $C(X)$, we define $r A$ to be $\{f+c \mid f \in A$ and $c \in R$ ). ( $R$ denotes the set of all real numbers, and we make the usual identification between the real number $c$ and the function which maps every $x \in X$ onto $c_{\text {.) }}$ We denote by $A^{u}$ the closure of $A$ in the uniform topology.

With each ideal $I$ in $C(X)$, we associate four subalgebras of $C(X), I$ itself, $I^{u}, r I$, and $r\left(I^{u}\right)$. (In [4], $r I$ and $r\left(I^{u}\right)$ were denoted by $(I)$ and $\left(I^{u}\right)$, respectively.) In this paper, we characterize the maximal ideals of $I^{u}, r I$, and $r\left(l^{u}\right)$ (the maximal ideals of $I$ were characterized in [4]) and then endow these sets of maxinal ideals with the hull-kernel topology. We then investigate the resulting structure spaces.

In $\$ 1$ we show that the prime and maximal ideals of $r l$ are the intersections of $r l$ respectively with the prime and maximal ideals of $C(X)$. This allows us to establish homeomorphisms between structure spaces of $r I$ and modifications of structure spaces of $C(X)$. We also show that the structure space of $r l$ is (homeomorphic to) the one-point compactification of the structure space of $l$.

In $\$ 2$ we discuss the prime and maximal ideals of the algebras $I^{u}$ and $r\left(I^{u}\right)$. We show that $I$ and $I^{u}$ have the same structure space and that $r I$ and $r\left(I^{u}\right)$ do also. In view of the fact that $r\left(I^{u}\right)$ is (isomorphic to) a ring of continuous functions (see $[4,5.6]$ ), it is thus established that every ideal in $C(X)$ is a real ideal in a subalgebra of $C(X)$ which has the same structure space as a ring of continuous functions. Results in $\$ \$ 1$ and 2 generalize certain results in [ 5 ].

Received by the editors August 1, 1972 and, in revised form, May 23, 1973.

AMS (MOS) subject classifications (1970). Primary 46E25, 54C40.

Key words and phrases. Rings of real-valued continuous functions, ideals, structure spaces, uniform closure. 
The significance of homeomorphic structure spaces of two rings of continuous functions is well known; namely $C(X)$ and $C(Y)$ have homeomorphic structure spaces if and only if $C^{*}(X)$ and $C^{*}(Y)$ are isomorphic. (These are the subrings of bounded functions in $C(X)$ and $C(Y)$ respectively.) In $\$ 3$ we establish necessary and sufficient conditions for two ideals in rings of continuous functions to have homeomorphic structure spaces.

In [2] and [3], the authors considered structure spaces of certain subalgebras of $C(X)$ called "algebras on $X . "$ In $\$ 4$, we discuss the relationships between the properties of an algebra on $X$ and the properties of the algebras considered in this paper.

Preliminaries and notations. For each $f \in C^{*}(X)$ there is a unique extension $\hat{f}$ from $\beta X$ into $R$. For each $f \in C(X)$, there is a unique extension $f^{*}$ from $\beta X$ into the two-point compactification of $R$. (See $[1,7.5]$ and $[4,2.4]$ for more about $f^{*}$.) If $f \in C^{*}(X)$, then $\hat{f}=f^{*}$.

For any commutative ring, a.collection of prime ideals endowed with the hull-kernel topology is called a structure space of the ring. The collection of all prime maximal ideals is referred to as the structure space of the ring. It is well known that $\beta X$ is (homeomorphic to) the structure space of either $C(X)$ or $C^{*}(X)$ and that $v X$ is the structure space of real maximal ideals of $C(X)$. (The reader is referred to 7.10 and 7.11 in [1] and [4, 2.3] for more about structure spaces.) The natural mapping $M \rightarrow M^{*}$, where $M^{*}=M^{u} \cap C^{*}(X)$, is a homeomorphism of the structure space of $C(X)$ onto the structure space of $C^{*}(X)$. (See $[1,7.11]$.)

Associated with each ideal $I$ in $C(X)$ is a space $X(I)$ and a fixed maximal ideal in $C(X(I))$ denoted by $F(I)$ with the property that $I^{u}$ is isomorphic to $F(I)$. (See $[4,5.6]$.)

If $f \in C(X)$, we shall let $(f)$ denote the principal ideal generated by $f$, and we let $Z(f)=\{x \in X \mid f(x)=0\}$. If $g \in C(X)$, then $f \vee g$ denotes the pointwise maximum of $f$ and $g$.

A subring $A$ of $C(X)$ is called a subalgebra of $C(X)$ if $A$ is closed under multiplication by constants. The structure space (of prime maximal ideals) of $A$ will be denoted by $\mu A$.

The symbol $\simeq$ is used to denote a homeomorphism between spaces, and $\approx$ is used to denote an isomorphism betwe en rings.

For the convenience of the reader we now list the main symbols associated with an ideal $I$ of $C(X)$.

$I^{u}=$ the uniform closure of $I$;

$I^{*}=I^{u} \cap C^{*}(X)$;

$r I=\{f+c \mid f \in I$ and $c \in R\}$;

$r\left(I^{u}\right)=\left\{f+c \mid f \in I^{u}\right.$ and $\left.c \in R\right\}$; 
$\mu I=$ the structure space of $I$;

$\Delta I=\bigcap\{Z(f) \mid f \in I\}$

$M(I)=\{M \mid M$ is a maximal ideal in $C(X)$ and $M \supseteq l\}$;

$P(I)=\{P \mid P$ is a prime ideal in $C(X)$ and $P \supseteq l\}$;

$X(I)=$ a space with the property that $C(X(I)) \approx r\left(I^{u}\right)$;

$F(I)=$ the real ideal in the space $X(I)$ isomorphic to $I^{u}$.

1. The structure space of $r l$. It is known $[4,3.6]$ that the maximal ideals of an ideal $I$ are precisely the intersections of $I$ with the maximal ideals of $C(X)$ not containing $l$. We now characterize the maximal ideals of the algebra $r l$.

1.1 Remark. Evidently, $I$ itself is a real maximal ideal of $r I$.

1.2 Lemma. If $M$ is a maximal ideal in $C(X)$, then $M \cap r I$ is a maximal ideal in $\mathrm{rl}$.

Proof. It is easy to show that for a proper ideal $J$ of $C(X), J \supseteq I$ if and only if $J \cap r I=I$. Thus if $M \supseteq I, M \cap r l$ equals $I$ and is a maximal ideal in $r l$. Now suppose $M \nsupseteq I$. Then there exist $m \in M$ and $i \in I$ with $m+i=1$. Assume $M \cap r l \subsetneq K \subseteq r l$, for some ideal $K$ of $r l$. Let $k \in K \backslash M$. Then there exist $m^{\prime} \in M$ and $b \in C(X)$ so that $b k+m^{\prime}=1$. Thus $i=i b k+i m^{\prime} \in K$. But $m=-i+1 \in M \cap$ $r I \subseteq K$; whence $1 \in K$ and $K=r l$.

We now proceed to establish the converse of 1.2.

1.3 Lemma. If $K$ is a proper prime ideal in $r l$, then $K=P \cap$ rl for some prime ideal $P$ in $C(X)$. Furthermore, $P$ is unique if $K \neq 1$.

Proof. From the multiplicative semigroup $G=(r l) \backslash K$. From Zorn's lemma, it follows that there exists a prime ideal $P$ in $C(X)$ so that $G \cap P=\varnothing$ and $P$ is maximal with respect to this property. (See $[1,0.16$.) We claim that $K=$ $P \cap r l$. It is easy to see that $K \supseteq P \cap r I$. Now if $P \supseteq l$, then $K=I=P \cap r l$, so assume $P \nsupseteq I$. We must show that $K \subseteq P$. To this end, let $k \in K$ and assume $k \notin P$. Let $J$ denote $P+k \cdot I$, an ideal in $C(X)$ which contains $P$ properly. Hence $J \cap G \neq \varnothing$, and there exists $p+k i \in G$, where $p \in P$ and $i \in I$. It follows that $p \in r l$ and hence $p \in K$. But then $p+k i \in K$, a contradiction. The uniqueness is evident.

1.4 Theorem. If $K$ is a maximal ideal in $r l$, then $K=M \cap r I$ for some maximal ideal $M$ in $C(X)$. Furtbermore, if $K \neq I$, then $M$ is unique.

Proof. By $[4,3.3] K$ is prime, and hence $K=P \cap r l$, for some prime ideal $P$ in $C(X)$. Letting $M$ be the maximal ideal containing $P$, the result follows.

We now consider some natural mappings between structure spaces of $I I$ and $C(X)$. We shall let $\mathcal{P}$ denote the space of prime ideals of $C(X), \mathscr{P}(r l)$ the space 
of prime ideals of $r l$ and $P(I)$ the set of all prime ideals in $C(X)$ which contain I. (Of course, $\mathscr{P}$ and $\mathscr{P}(r I)$ are endowed with the hull-kernel topology.)

1.5 Lemma. $\mathcal{P} \backslash P(I)$ is bomeomorpbic to $\mathcal{P}(r I) \backslash I I$.

Proof. Consider the natural mapping $P \rightarrow P \cap r l$ and denote it by $\phi$. Clearly $\phi$ is one-to-one and onto. Let $s \in r l$ and consider $E(s)=\{K \mid K \in \mathscr{P}(r I) \backslash I\}$ and $s \in K\}$, a basic closed set in $\mathscr{P}(r I) \backslash\{I\}$. Then $\phi^{-1}(E(s))=\{P \in \mathcal{P} \backslash P(I) \mid s \in P\}$ a basic closed set in $\mathscr{P} \backslash P(I)$. On the other hand, if $f \in C(X)$, and $E(f)=$ $\{P \in \mathcal{P} \backslash P(I) \mid f \in P\}$, a basic closed set in $\mathscr{P} \backslash P(I)$, then $\phi(E(f))=\{P \cap r I \mid P \cap$ $r I \supseteq f \cdot I\}$, a closed set in $P(r I) \backslash\{I\}$.

We now extend the mapping $\phi$ to obtain a mapping onto all of $\mathcal{P}(r l)$. The preimage of $I$ is $P(I)$ shrunk to a point. Specifically, we let $\mathscr{P}^{\prime}=[\mathcal{P} \backslash P(I)] \cup\{\alpha\}$ where a neighborhood of $a$ is a set of the form $\{a\} \cup[W \backslash P(I)]$ where $W$ is open in $\mathcal{P}$ and $W \supseteq P(I)$. (For $P \in \mathcal{P} \backslash P(I)$, a neighborhood of $P$ is a neighborhood in the relative topology.)

1.6 Theorem. Let $\psi: \mathscr{P}^{\prime} \rightarrow \mathscr{P}(r I)$ be defined by $\psi(P)=P \cap$ rl if $P \nsupseteq I$, and $\psi(a)=I$. Then $\psi$ is a bomeomorphism of $\mathcal{P}^{\prime}$ onto $\mathcal{P}(r l)$.

Proof. Clearly $\psi$ is one-to-one and onto $\mathscr{P}(r I)$, and by virtue of 1.5 , it suffices to show that $\psi$ is continuous at $a$ and $\psi^{-1}$ is continuous at 1 . To this end, let $V$ be a basic neighborhood of $\psi(\alpha)$ in the space $\mathcal{P}(r l)$. Then $V=$ $\sim\{K \in \mathcal{P}(r l) \mid s \in K\}$ for some $s=i+c \in r l$. Since $I \in V, c \neq 0$. Let $W=$ $\sim\{P \in \mathcal{P} \mid s \in P\}$, an open set in $\mathcal{P}$.

It is easily seen that $W \supseteq P(I)$, for if $P \supseteq I$, and $P \notin W$, it would follow that $s \in P$, a contradiction. Thus the set $\{a\} \cup[W \backslash P(I)]$ is open in $\mathcal{P}^{\prime}$, and it follows that its image under $\psi$ is contained in $V$.

We now show that $\psi^{-1}$ is continuous at $I$. Let $\{\alpha\} \cup[W \backslash P(I)]$ be a neighborhood of $a$ in $\mathcal{P}^{\prime}$, with $W=\sim\{P \in \mathcal{P} \mid P \supseteq J\}$ for some ideal $J$ in $C(X)$. Since $W \supseteq P(I)$, it follows that $I+J=C(X)$, and hence there exist $i \in I$ and $j \in J$ so that $i+j=1$. Letting $V$ denote the open set $\sim\{K \in \mathcal{P}(r l) \mid j \in K\}(j \in r l)$ it follows that $\psi^{-1}(V) \subseteq\{a\} \cup[W \backslash P(I)]$.

1.7 Remark. If we shrink $M(I)$ to a point in the space of maximal ideals in $C(X)$, then the resulting space will be homeomorphic to $\mu(r I)$. (The argument would be the same as 1.5 and 1.6 above.) It then follows that $\mu(r I)$ is homeomorphic to $(\mu I)^{*}$, the one-point compactification of $\mu l$. (See $[4,3.9]$.)

1.8 Remark. If we denote the set of all real maximal ideals containing $I$ by $R(I)$ (possibly $R(I)=\varnothing$ ), then the structure space of real ideals of $r l$ is homeomorphic to $(u X \backslash R(I)) \cup\{a\}$ where a neighborhood of $\alpha$ is a set of the form $\{a\} \cup$ $[W \backslash R(I)]$ for some $W$ open in $v X$ with $W \supseteq R(I)$. 
Of course, the albebra $r l$ will not in general be (isomorphic to) a ring of continuous functions. Indeed since $I$ is a real ideal in $r l$, it follows from $[4,5.7]$ that $r I$ is a ring of continuous functions if and only if $I$ is uniformly closed.

In view of the fact that $\left(f_{1}+c_{1}\right) \vee\left(f_{2}+c_{2}\right)=\left(f_{1} \vee f_{2}\right)+\left(c_{1} \vee c_{2}\right)$ where $f_{j} \in C(X)$ and $c_{j}$ are real numbers, it follows that $r l$ is a $\Phi$-algebra (see [s]) if and only if $f \vee g \in I$ whenever $f$ and $g$ are members of $I$. In particular, $r I$ is a $\Phi$-algebra whenever $l$ is absolutely convex in $C(X)$. (An ideal $I$ is said to be absolutely convex in $C(X)$ if $f \in I$ and $g \in C(X)$ with $|g| \leq|f|$ imply that $g \in I$.)

2. The albebras $I^{\mu}$ and $r\left(I^{u}\right)$. We now proceed to characterize the maximal ideals of $\mu^{\mu}$ and $r\left(I^{u}\right)$. We recall that in $[4,5.6]$ it was shown that $r\left(I^{\mu}\right)$ is (isomorphic to) a ring of continuous functions. (Indeed, $r\left(I^{u}\right)$ is in a sense the smallest ring of continuous functions in which $l$ is an ideal.)

2.1 Remark. $I^{u}$ is a real maximal ideal in $r\left(I^{u}\right)$.

2.2 Lemma. If $s \in r\left(I^{u}\right)$ with $s(x) \geq \delta>0$ for all $x \in X$, then $(1 / s) \in r\left(I^{u}\right)$.

Proof. Suppose $s=f+c$ where $f \in I^{u}$ and $c \in R$, and $s(x) \geq \delta>0$ for all $x \in X$. It is easily seen that $c \neq 0$, and we then have $1 / s=-f / d(s)+1 / c$. We claim that $f /(f+c) \epsilon I^{u}$. To see this, let $\epsilon>0$ be given, and consider $\epsilon^{\prime}=\delta \cdot \epsilon$. Then there exists $i \in I$ with $|f-i|<\epsilon^{\prime}$, whence

$$
\left|\frac{f}{f+c}-\frac{i}{f+c}\right|<\frac{\epsilon^{\prime}}{|f+c|} \leq \frac{\epsilon^{\prime}}{\delta}=\epsilon .
$$

2.3 Lemma. If $M$ is a maximal ideal in $C(X)$ with $M \nsupseteq I$, then $M \cap r\left(I^{u}\right)$ is a maximal ideal in $r\left(I^{\mu}\right)$.

Proof. The argument is the same as 1.2.

2.4 Remark. If $M \supseteq I$, it is possible that $M \cap r\left(I^{\mu}\right) \subsetneq l^{\mu}$, and hence $M \cap$ $r\left(l^{u}\right)$ is not maximal (for example, if $I$ is a hyperreal maximal ideal and $M=I$ ).

2.5 Remark. It is possible to have prime ideals in $r\left(I^{u}\right)$ which are not of the form $P \cap r\left(I^{u}\right)$ for $P$ prime in $C(X)$. As a simple example, the ideal $I^{u}$ itself $m$ ay not be of this form. We can say, however

2.6 Lemma. If $K$ is a prime ideal in $r\left(I^{u}\right)$ and $K \nsupseteq l$, then $K=P \cap r\left(I^{u}\right)$ for a unique prime ideal $P$ in $C(X)$.

Proof. The argument is essentially the same as 1.3 .

2.7 Theorem. Let $K$ be a maximal ideal of $r\left(I^{\mu}\right)$. If $K \nsupseteq I$, then $K=M \cap$ $r\left(I^{u}\right)$ for a unique maximal ideal $M$ in $C(X)$. If $K \supseteq I$, then $K=I^{u}$. 
Proof. The first part of the theorem is evident. For the second part, suppose $K \supseteq I$, and let $k \in K$, say $k=f+c$ where $f \in I^{u}$ and $c \in R$. Assume $c>0$. Then for some $i \in l,|f-i|<c / 3$. Since $i \in K$, it follows that $f+c-i \in K$. But $(f+c-i)(x)>2 c / 3$ for all $x \in X$, and this contradicts 2.2. Similarly, if $c<0$ we arrive at a contradiction, so we must have $c=0$ and $K \subseteq l^{u}$.

We now consider the maximal ideals of the algebra $I^{u}$. We shall make use of the fact that $r\left(I^{u}\right)$ is isomorphic to $C(X(I))$ and that the isomorphism takes $I^{u}$ onto $F(I)$.

2.8 Theorem. Let $K$ be a maximal ideal in $I^{u}$. Then $K=M \cap I^{u}$ for some unique maximal ideal $M$ in $C(X)$ with $M \nsupseteq I$.

Proof. Let $\xi$ denote the isomorphism of $r\left(I^{u}\right)$ onto $C(X(I))$. Since $K$ is a maximal ideal in $I^{u}$, it follows that $\xi(K)=F(l) \cap M^{\prime}$ where $M^{\prime}$ is maximal in $C(X(I))$. But then $M^{\prime}=\xi\left[M \cap r\left(I^{u}\right)\right]$ (clearly $M^{\prime} \neq \xi\left(I^{u}\right)$ ) and it follows that $K=$ $M \cap I^{u}$. Now, as sume $M \supseteq I$, and let $f \in I^{u} \backslash K$. From the maximality of $K$, it follows that $K+f \cdot l^{u}=I^{u}$, whence $f=k+f g$ for some $k \in M \cap I^{u}$ and $g \in I^{u}$. But this implies that $k=f(1-g) \in M$, and hence $1-g \in M$. Since $M \supseteq l, g \in M^{u}$, a contradiction.

2.9 Corollary. The ideal $I$ in $I^{u}$ is not contained in a maximal ideal of $I^{u}$.

Proof. Follows directly from 2.8 above.

2.10 Lemma. If $M$ is maximal in $C(X)$ and $M \nsupseteq l$, then $M \cap I^{u}$ is a maximal ideal in $I^{u}$. If $M \supseteq I$, then $M \cap I^{u}$ is not a maximal ideal in $I^{u}$.

Proof. The argument for the first part is the same as 1.2. The second part follows from 2.9.

2.11 Corollary. $\mu I$ is bomeomorphic to $\mu\left(I^{\mu}\right)$.

Proof. The natural mapping is a homeomorphism.

2.12 Remark. It is easily seen that the natural mapping $M \cap r l \rightarrow M \cap r\left(l^{u}\right)$, for $M \nsupseteq l$, and $I \rightarrow I^{u}$ is a homeomorphism of $\mu(r l)$ onto $\mu\left(r\left(I^{u}\right)\right)$, and $\mu\left(r\left(I^{u}\right)\right)$ is the structure space of a ring of continuous functions. We thus have that every ideal in a ring of continuous functions is a real ideal in an algebra whose structure space is homeomorphic to a structure space of a ring of continuous functions.

2.13 Remark. $(r I)^{u}=r\left(I^{u}\right)$.

Proof. Let $s \in(r l)^{u}$. Then there is a sequence $\left\langle s_{n}\right\rangle$ in $r l$, say $s_{n}=i_{n}+c_{n}$ where $i_{n} \in I$ and $c_{n} \in R$, which converges to $s$. We claim that the sequence $\left\langle c_{n}\right\rangle$ is a Cauchy sequence. To see this, consider $\epsilon>0$. There exists a positive 
integer $N$, so that $\left|s(x)-\left(i_{n}(x)+c_{n}\right)\right|<\epsilon / 2$ for all $n>N$ and all $x \in X$. Let $n$ and $m$ be any positive integers greater than $N$, and let $t \in X$ so that $i_{n}(t)=$ $i_{m}(t)=0$. We then have $\left|s(t)-c_{n}\right|<\epsilon / 2$ and $\left|s(t)-c_{m}\right|<\epsilon / 2$, from which it follows that $\left|c_{m}-c_{n}\right|<\epsilon$. Let $c$ denote $\lim _{n \rightarrow \infty} c_{n}$. It then follows that the sequence $\left\langle i_{n}\right\rangle$ converges to a function $f \in I^{\mu}$, and $s=f+c$. Conversely, if $s=$ $f+c \in r\left(l^{u}\right)$ then, given $\epsilon>0$, there exists $i \in I$ so that $|f-i|<\epsilon$. But $|f-i|=$ $|(f+c)-(i+c)|$, and $i+c \epsilon r l$.

The above remark generalizes [ $5,3.8]$.

2.14 Remark. The algebras $r l$ and $r\left(I^{u}\right)$ cannot be (ring-) isomorphic unless they are identical. To see this, it suffices to observe that any isomorphism would take $I$ onto $I^{u}$ which would imply their equality by $[4,4.8]$.

3. Structure spaces of ideals. The structure space of an ideal is not usually a compact space. Indeed

3.1 Remark. For any ideal $I$, the following are equivalent.

(1) $\mu I$ is compact.

(2) $M(I)$ is open (and closed) in $\beta X$.

(3) $I$ is the principle ideal generated by an idempotent.

Proof. The equivalence of (1) and (2) follows from the fact that $\mu I \simeq$ $\beta X \backslash M(I)$ (see $[4,3.9])$.

$(2) \Rightarrow(3)$ If $M(I)$ is open, then $\sim M(I)=\{M \mid M \supseteq J\}$ for some ideal $J$ in $C(X)$. It follows that $I \cap J=\{0\}$ and $I+J=C(X)$, and hence there exist $i \in I$ and $j \in J$ with $i+j=1$. Thus $I=(i)$ and $i^{2}=i$.

(3) $\Rightarrow(2)$ If $I=(i)$ and $i^{2}=i$, let $J=(i-1)$. Then $\sim M(I)=\{M \mid M \supseteq J\}$.

However, the structure space of $I$ is always locally compact, and its onepoint compactification is homeomorphic to $\mu\left(r\left(I^{\mu}\right)\right)$, a Stone-Čech compactification $(\beta[X(I)])$. (It is also true that any $\beta X$ is the one-point compactification of a $\mu I$; simply take $I$ to be a maximal ideal in $C(X)$.) Thus $\mu I$ is in a sense a large space, in that it lacks only one point from being a Stone-Čech compactification.

We now wish to show to what extent the structure space of $I$ determines $I$; specifically, when are $\mu I$ and $\mu J$ homeomorphic?

We begin with a mapping from the set of ideals in $C(X)$ into the set of ideals of $C^{*}(X)$. For an ideal $l$ in $C(X)$, we denote $I^{u} \cap C^{*}(X)$ by $l^{*}$. Note that this * mapping is the usual one from the set of maximal ideals in $C(X)$ onto the set of maximal ideals in $C^{*}(X)$. (See $[4,2.4]$.)

3.2 Lemma. $I^{*}=\left\{f \in C^{*}(X) \mid Z(\hat{f}) \supseteq M(I)\right\}$.

Proof. Follows from $[4,5.1]$ and the fact that $f^{*}=\hat{f}$ for bounded functions.

3.3 Remark. The ${ }^{*}$ mapping is onto the set of full ideals of $C(\beta X)$. (An ideal is said to be full in $C(Y)$ if it is of the form $\{f \in C(Y) \mid Z(f) \supseteq G\}$ for some closed set $G$ in $Y$.) 
3.4 Lemma. $\mu I$ is bomeomorphic to $\mu\left(I^{*}\right)$.

Proof. The mapping ${ }^{*}$ is a homeomorphism of the structure space of $C(X)$ onto the structure space of $C^{*}(X)[1,7.11]$, and clearly $M(I)$ will be mapped onto the set of maximal ideals in $C^{*}(X)$ which contain $I^{*}$.

3.5 Lemma. $I^{*}$ is isomorphic to the set of continuous real-valued functions on $\mu(r l)$ which vanisb at $I$.

Proof. Let $M_{I}$ denote $\{g \in C(\mu(r I)) \mid g(I)=0\}$ and for each $f \in I^{*}$, let $\bar{f}$ be the function on $\mu(r l)$ defined by $\bar{f}(M \cap r I)=\hat{f}(M)$ for $M \nsupseteq I$ and $\bar{f}(I)=0$. By virtue of the homeomorphism of $\mu(r I)$ into $\beta X$, it follows that $\bar{f}$ is continuous at $M \cap r l$ for any $M \nsupseteq I$. To see that $\bar{f}$ is continuous at $I$, consider $\epsilon>0$. Let $W=$ $\hat{f}^{-1}(-\epsilon, \epsilon)$, an open set in $\beta X$, and hence $W=\sim\{M \mid M \supseteq J\}$ for some ideal $J$ in $C(X)$. For any $M \supseteq l, \hat{f}(M)=f^{*}(M)=0$ by $[4,5.1]$, from which it follows that there exist $i \in I$ and $j \in J$ with $i+j=1$. Consider $U=\sim\{K \in \mu(r I) \mid j \in K\}$, a neighborhood of $I$ in $\mu(r I)$. Clearly, for any $M \cap(r I) \in U, M \in W$, and hence $\bar{f}(M) \epsilon(-\epsilon, \epsilon)$.

For any $g \in M_{I}$, define $\hat{f}: \beta X \rightarrow R$ by $\hat{f}(M)=g(M \cap r l)$ for $M \nsupseteq I$ and $\hat{f}(M)=0$ for $M \supseteq I$. Then $f \in I^{*}$ and $\bar{f}=g$. ( $f$ is the restriction of $\hat{f}$ to $\left.X.\right)$

Using the fact that " ${ }^{\wedge}$ is an isomorphism of $C^{*}(X)$ onto $C(\beta X)$, it follows easily that the mapping $f \rightarrow \bar{f}$ is an isomorphism of $I^{*}$ onto $M_{I^{\bullet}}$

3.6 Theorem. $\mu I \simeq \mu J$ if and only if $I^{*} \approx J^{*}$.

Proof. If $\mu l \cong \mu J$, then their one-point compactifications are homeomorphic. Thus, $\mu(r I) \simeq \mu(r J)$, and this homeomorphism takes the point $I$ onto the point $J$. It follows that $M_{l}$ and $M_{J}$ (as in the notation of the proof of 3.5) are isomorphic; whence $I^{*} \approx J^{*}$ by 3.5 .

Conversely if $I^{*} \approx J^{*}$, then $\mu\left(I^{*}\right) \simeq \mu\left(J^{*}\right)$, which, by 3.4 , yields the required result.

3.7 Corollary. If $I^{u} \approx J^{u}$, then $I^{*} \approx J^{*}$.

Proof. By 2.11 , the hypothesis implies that $\mu I \simeq \mu \mathrm{J}$. The result then follows from 3.6 above.

3.8 Corollary. $\mu l \cong \mu J$ if and only if $[F(I)]^{*} \approx[F(J)]^{*}$.

Proof. Evidently the mapping $l^{u} \rightarrow F(I)$ described in $\$ 5$ of [4] preserves bounded functions, and so does its inverse. Thus $I^{*} \approx[F(D)]^{*}$ and the result follows by 3.6.

3.9 Remark. It is possible for $\mu I \simeq \mu J$ without $I^{u} \approx J^{u}$. For example, let $X$ be any realcompact space which is not $\beta$-fixed (see $[4,6.12])$ and let $H$ be a 
homeonorphi of $\beta X$ onto itself which takes a real ideal $M_{1}$ onto a hyperreal ideal $M_{2}$. Then $\left[\beta X \backslash\left\{M_{1}\right\}\right] \simeq\left[\beta X \backslash\left\{M_{2}\right\}\right.$, i.e. $\mu M_{1} \simeq \mu M_{2}$, but certainly $\left(M_{1}\right)^{u}$ and $\left(M_{2}\right)^{u}$ are not isomorphic.

3.10 Remark. Theorem 3.6 tells us that the structure space of $I$ in general tells us very little about the algebraic properties of the ideal $I$, since vastly different ideals can have the same uniform closures. Even with real ideals, homeomorphism of structure spaces does not necessarily imply isomorphism of the ideals. For example, consider $M=\{f \in C(N) \mid f(1)=0\}$ and $M^{\prime}=\{f \in C(\Sigma) \mid f(1)=0\}$ where $N$ is the space of natural numbers and $\Sigma$ is the space of Exercise $4 M$ in [1]. Then $\mu M$ and $\mu M^{\prime}$ are homeomorphic (since $\beta N \simeq \beta \Sigma$ ) but certainly $M$ and $M^{\prime}$ cannot be isomorphic (since $C(N)$ and $C(\Sigma)$ are not isomorphic).

Homeomorphism of real structure spaces of ideals does not seem to tell much about the algebraic structure of the ideals either. For example, let $Q$ denote the space of rationals and let $X$ denote the rationals with $\{0\}$ open. Then $M=$ $\{f \in C(Q) \mid f(0)=0\}$ and $M^{\prime}=\{f \in C(X) \mid f(0)=0\}$ have the same structure space of real ideals.

4. Algebras on $X$. In [2], the author defines an algebra on $X$ to be a subalgebra $A$ of $C(X)$ with the following properties: (i) $A$ contains the constant functions. (ii) $A$ is uniformly closed. (iii) If $F$ is closed in $X$ and $x \in X \backslash F$, then there exists $f \in A$ so that $f(x) \neq 0$ and $f(F)=0$. ( $A$ separates points and closed sets.) (iv) If $f \in A$ with $Z(f)=\varnothing$, then $1 / f \in A$. ( $A$ is closed under inversion.)

Of the four algebras considered here $\left(I, l^{u}, r l, r l^{u}\right)$, none will be an algebra on $X$ in general. We now discuss in a series of remarks and examples these algebras with respect to properties (iii) and (iv) above, since the first two properties involve only trivial considerations.

4.1 Remark. $r I$ is closed under inversion in $C(X)$.

Proof. Let $f+c \in r I(f \in I$ and $c \in R)$ with $Z(f+c)=\varnothing$. Clearly $c \neq 0$, and we have $1 /(f+c)=g+1 / c$ where $g=((-1 / c) /(f+c)) \cdot f \in I$.

We note that the above remark generalizes $[5,3.2]$.

In general, $r\left(I^{\mu}\right)$ is not closed under inversion in $C(X)$. This may seem somewhat surprising in view of the fact that $r\left(l^{u}\right)$ is (isomorphic to) a ring of continuous functions.

4.2 Example. Let $M$ be a hyperreal maximal ideal in $C(X)$. Then there exists a unit $b \in M^{u}[1,7.9(b)]$. If $1 / b \in r\left(M^{u}\right)$, we would have $1 / b=m+c$, $m \in M^{u}$ and $c \in R$, whence $1=b \cdot m+b \cdot c \in M^{u}$, a contradiction.

4.3 Remark. An ideal $I$ determines the topology on $X$ (separates points and closed sets) if and only if $I$ is a free ideal. 
Proof. Assume $I$ is a free ideal, and let $K$ be closed in $X$ with $x \in X \backslash K$. There exists $f \in I$ with $f(x) \neq 0$ and there exists $g \in C(X)$ with $g(x) \neq 0$ and $g(K)=0$. Thus $f \cdot g \in I$ which separates $x$ from $K$. If $I$ is a fixed ideal, then no point in $\Delta I$ can be separated from a closed set not containing that point.

The algebra $r l$ clearly cannot determine the topology on $X$ if $\Delta I$ has at least two points, and $r I$ does determine the topology on $X$ if $\Delta I=\varnothing$. If $\Delta I$ consists of precisely one point, then $r l$ may or may not determine the topology.

4.4 Example. Let $X$ be the real line and $I=\{f \in C(X) \mid f(0)=0$ and $f$ is eventually zero\}. Let $K$ be the closed set $\{1,2,3,4, \cdots\}$. Then $0 \notin K$, but no function in $r l$ can separate 0 from $K$.

4.5 Remark. The isomorphic image $r \bar{l}$ of $r l$ under the natural isomorphism of $r\left(I^{u}\right)$ onto $C(X(I))$ (see $\left.[4,5.4]\right)$ determines the topology on $X(I)$.

Proof. Since $\Delta \bar{l}=\{F\}$, we have the ambiguous case described above. Let $x \in X(I)$ and $U$ an open set in $X(I)$, with $x \in U$. If $x$ is different from the point $F$, then $x \in X \backslash(F \cap X)$, and hence there is an $f \in I$ with $f(x) \neq 0$. Let $g \epsilon$ $C(X(I))$ with $g(x) \neq 0$ and $g(\sim U)=0$. Then $\bar{f} \cdot g$ separates $x$ from $U$. If $x$ is $F$ itself, then $U=\{F\} \cup[V \cap(X \backslash F)]$ where $V$ is open in $\beta X$ and $V \supseteq F$. By the normality of $\beta X$, we can find an open set $W$ and a function $\hat{b} \in C(\beta X)$ so that $F \subseteq$ $W \subseteq \operatorname{cl}_{\beta X} W \subseteq V$ and $\hat{b}(W)=0$ and $\hat{b}(\sim V)=1$. Also, there exists $\hat{k} \in C(\beta X)$ with $\hat{k}(F)=0$ and $\hat{k}(W)=1$, and hence $\hat{b}=\hat{b} \cdot \hat{k}$. As usual we denote the restrictions of $\hat{b}$ and $\hat{k}$ to $X$ by $b$ and $k$ respectively. We consider the functions $\bar{b}, \bar{k}$ on the space $X(I)$. It is easily seen that $\bar{b}=\bar{b} \cdot \bar{k}$ and that $\bar{k}(F)=0$. Hence $\bar{b} \in m F(I)$, whence $b \in I$. Since $\bar{b}(F)=0$ and $\bar{b}(\sim U)=1$, the result is established.

4.6 Remark. $r \bar{I}$ is closed under inversion in $C(X(I))$. (The proof is the same as Remark 4.1.) If $r \bar{I}$ is uniformly closed, then $r \bar{l}=C(X(I))$ by 2.13 , and only in this case can $r \bar{l}$ be an algebra on $X(I)$.

If $A$ is an algebra on $X$, then the structure space of maximal ideals $\mu A$ (denoted by $H\left(A^{*}\right)$ in [2] and [3]) is a compactification of $X$. Among its properties are

(i) $A \cap C^{*}(X)=C(\mu A)$;

(ii) $A=E(\mu A)=$ the ring of continuous functions from $\mu A$ into the two-point compactifications of the reals. (See $[2,1.2]$.)

Of course, results such as the above do not hold in general for the algebras considered here.

\section{REFERENCES}

1. L. Gillman and M. Jeri son, Rings of continuous functions, University Series in Higher Matho, Van Nostrand, Princeton, N. J., 1960. MR 22\#6994.

2. A. W. Hager, On inverse-closed subalgebras of $C(X)$, Proc. London Math. Soc. (3) 19 (1969), 233-257. MR 39\#6261. 
3. J. R. Isbell, Algebras of uniformly continuous functions, Ann. of Math. (2) 68 (1958), 96-125. MR 21 \# 2177.

4. D. Rudd, On isomorphisms between ideals in rings of continuous functions, Trans Amer. Math. Soc. 159 (1971), 335-353. MR 44 \# 806.

5. - An example of a -algebra whose uniform closure is a ring of continuous functions, Fund. Math. 77 (1972), 1-4. 23508

DEPARTMENT OF MATHEMATICS, OLD DOMINION UNIVERSITY, NORFOLK, VIRGINIA 